\title{
Acute Effects of Static Upper and Lower Body Vibration Exercise on Arterial Compliance in Male Subjects
}

Joe-Angel Lopez, Guillermo Perez, Murat Karabulut. University of Texas at Brownsville, Brownsville, TX.

PURPOSE: To examine the responses in arterial compliance (AC) of the small and large arteries, and systemic vascular resistance to static whole body vibration (WBV) exercise with blood flow restriction (BFR). METHODS: Eight male participants $(\mathrm{age}=22.6 \pm 2.2)$ randomly performed two sessions of static upper body and two sessions of static lower body exercise on a vibration platform with and without applied BFR. Prior to testing, subjects laid in a supine position while resting large arterial compliance (LAC), small arterial compliance (SAC), and systemic vascular resistance (SVR) were assessed using pulse wave analysis. During BFR trials, BFR cuffs were placed at the most proximal area of the thighs and arms. The BFR cuffs were then inflated to a target restrictive pressure (TRP) of $100 \mathrm{mmHg}$ for upper body and to a TRP determined by multiplying the systolic blood pressure by 1.44 for lower body. Tests were performed on a vibration platform set at $30 \mathrm{~Hz}$ at either a static pushup or squat position and held at an angle of $110^{\circ}$ at the elbows and knees. Exercises consisted of eight 45-s sets with a 45-s rest between each set for upper body, and for ten 1-min sets with a 1-min rest between each set for lower body. LAC, SAC, and SVR were assessed post-exercise at 10-min, 20-min, and 40-min. RESULTS: A main time effect $(p<0.02)$ was seen for LAC. However, post hoc comparisons did not find a significant difference. When BFR was applied, a trend was seen for decreases in SAC $(p<0.09)$. No condition effect was seen for SVR, but there was a significant time main effect for SVR from 10 min to 40 min post exercise $(p<0.02)$. CONCLUSION: Since exercises with BFR are physiologically more demanding due to lower venous return, possible elevation in epinephrine levels may account for trend seen for decreased SAC when BFR was applied. Furthermore, since exercise was performed at a static position, mechanoreceptors of vessel walls may have not been sufficiently stimulated to significantly affect SAC and LAC. However, the decrease in SVR observed 10 min following exercise, suggests that vasodilation took place resulting in decreases in systemic resistance against blood flow. Additional research is needed to test how a dynamic exercise protocol or varying intensities combined with BFR affect AC.

Key Words: Arterial Compliance, Blood Flow Restriction, Whole Body Vibration. 